\title{
Safety Rounds: A Patient Safety Initiative
}

\author{
Heather Kertland, Salma Satchu, Clarence Chant, Jill Garland, and Elaine Tom
}

\section{INTRODUCTION}

$T_{\mathrm{r}}^{\mathrm{h}}$ he reporting of medication safety incidents is an important responsibility of hospital pharmacists. The authors had the impression that within the Department of Pharmacy at their institution, medication safety incidents, including near misses, were not being reported or were being reported reluctantly. Computerized prescriber order entry (CPOE) and electronic medication administration records (eMARs) were soon to be implemented at this hospital. With the planned changes in pharmacy and medication workflows, it was expected that additional safety concerns would arise. We used an established safety program, the Comprehensive Unit-based Safety Program (CUSP), ${ }^{1}$ to identify, implement, and measure a safety initiative to see whether we could improve the culture of safety within the department.

\section{DESCRIPTION OF PROGRAM}

St Michael's Hospital is a university-affiliated tertiary care hospital with 463 inpatient beds in Toronto, Canada. The CUSP was chosen as the model for the Department of Pharmacy's safety initiative, because it calls for front-line staff to identify the safety issue that will be addressed and its possible solutions; in addition, CUSP requires that the impact of the intervention be measured. The CUSP model is a 6-step quality improvement program, as described in Table 1. This implementation and evaluation project involved both inpatient and hospital-affiliated clinic pharmacists; however, pharmacists working in the outpatient community pharmacy of the hospital were excluded.

Step 1 of CUSP involved conducting a baseline assessment (in May 2011) to allow subsequent measurement of the impact of the intervention. The Hospital Survey on Patient Safety Culture, ${ }^{2}$ a validated survey instrument, was used to assess 7 unit-level and 3 hospital-level aspects of safety culture and to determine an overall safety grade. For each statement in the survey, the number of responses of "agree" and "strongly agree" was used to calculate the positive response rate. To determine the overall safety grade, respondents were asked to choose 1 of 5 responses, ranging from "excellent" to "failing". The survey was disseminated electronically through the SurveyMonkey online survey tool at 2 time points: baseline (step 1 of CUSP) and 1 month after completion of CUSP (step 6). The timing of the second survey, at a point when the intervention had been in place for 4 months, was chosen because we did not wish concurrent medication system changes to affect the results. All pharmacists who had worked in the department both before and after CUSP implementation were invited to complete the surveys. Completion of the survey implied consent to participate in the study. The protocol was approved by the St Michael's Hospital Research Ethics Board.

As step 2 of CUSP, the institution's medical director of quality and safety provided 2 educational sessions to pharmacists on the topic of the culture of safety and the institution's safety initiatives. After these sessions, pharmacists were asked to complete another online survey (SurveyMonkey) to identify their top 3 safety concerns and their preferred methods of disseminating any safety information (step 3). Responses were collated by themes. The top safety concern identified in this survey was the possibility that new types of errors might be introduced by implementation of the CPOE/eMAR system. The preferred method of communicating information about the occurrence of errors related to the new CPOE/eMAR system was a group session that would provide an open forum for discussion of the errors and their potential resolution. Hence, "safety rounds" became the intervention identified for implementation and measurement. The initiative was endorsed by the director of pharmacy (step 4).

The safety rounds were modelled after "Pharmacy Improving Patient Safety" rounds at the London Health Sciences Centre (London, Ontario), ${ }^{3}$ although the structure and follow-up differed. Our safety rounds were hour-long sessions held every 2 weeks (step 5). All pharmacists were encouraged to attend during the first 4 months of the program. To best foster safety improvement, ground rules were established and agreed upon by participants with regard to confidentiality, shared learning, and 


\section{Table 1. Steps in the Comprehensive Unit-Based Safety Program ${ }^{1}$}

\begin{tabular}{|c|c|c|}
\hline Step & Activity & Site-Specific Details \\
\hline 1: Measurement & Conduct cultural survey & $\begin{array}{l}\text { Collected baseline data using the Hospital } \\
\text { Survey on Patient Safety Culture. }\end{array}$ \\
\hline 2: Education & $\begin{array}{l}\text { Provide training in the science } \\
\text { of safety }\end{array}$ & $\begin{array}{l}\text { Medical director of quality and safety } \\
\text { delivered } 2 \text { lectures on the culture and } \\
\text { science of safety to pharmacy staff. }\end{array}$ \\
\hline 3: Engagement & $\begin{array}{l}\text { Identify safety concerns and } \\
\text { interventions }\end{array}$ & $\begin{array}{l}\text { Pharmacists were asked to identify the top } \\
3 \text { patient safety concerns within the } \\
\text { Department of Pharmacy and to identify } \\
\text { methods by which department members } \\
\text { could work together to resolve safety } \\
\text { issues. The project team compiled results } \\
\text { and identified safety rounds as the } \\
\text { intervention. }\end{array}$ \\
\hline 4: Endorsement & Garner senior executive support & $\begin{array}{l}\text { Proposed safety rounds were introduced } \\
\text { at the monthly pharmacist meeting. The } \\
\text { proposed intervention received support } \\
\text { from the director of pharmacy. }\end{array}$ \\
\hline 5: Intervention & Deliver program & $\begin{array}{l}\text { Safety rounds were held every } 2 \text { weeks } \\
\text { for } 4 \text { months (total of } 8 \text { rounds, } 1 \text { h each). } \\
\text { Meeting minutes taken during each } \\
\text { session were shared with participants. }\end{array}$ \\
\hline 6: Measurement & $\begin{array}{l}\text { Document outcome of } \\
\text { intervention }\end{array}$ & $\begin{array}{l}\text { Program was evaluated, using the Hospital } \\
\text { Survey on Patient Safety Culture, } 1 \text { month } \\
\text { after completion of step 5, and results } \\
\text { were compared with baseline data. }\end{array}$ \\
\hline
\end{tabular}

the nonpunitive nature of the rounds. The safety rounds were facilitated by the Department of Pharmacy's professional practice leader (E.T.). Before rounds, actual or near misses and associated safety concerns were identified by pharmacists by means of the hospital's incident tracker or were reported directly to the professional practice leader. During rounds, each case or incident was presented, along with a root-cause analysis, if applicable. Participants then discussed the case, to validate the findings of the root-cause analysis and to explore potential process and practice changes that could prevent or minimize recurrence. A total of 8 safety rounds were conducted over 4 months. On average, 2 to 4 incidents or topics were discussed at each session. The average attendance was 21 pharmacists (range 8 to 29).

\section{FINDINGS}

Before the intervention, 33 (72\%) of 46 eligible pharmacists completed the Hospital Survey on Patient Safety Culture; after the intervention, 14 (50\%) of 28 eligible pharmacists did so. The lower number of eligible respondents for the post-intervention survey was due to staff turnover; all responses submitted for each survey were included in the analysis. The demographic characteristics for the 2 groups were similar with respect to age, experience, and training (data not reported). There was no change in rating for the domain for "overall perception of safety" (46\% before the intervention versus $44 \%$ after the intervention). Several domains showed a substantial increase in positive rating (e.g., teamwork within the unit and feedback about errors), while for others there was a decrease in positive rating (e.g., hospital management support for patient safety, teamwork across units, and handoffs and transitions) (Figure 1). All respondents rated the overall safety grade for the department as excellent, very good, or acceptable both before and after the intervention (before the intervention: excellent $9 \%$, very good $47 \%$, acceptable $44 \%$; after the intervention: very good $53 \%$, acceptable $47 \%$ ).

\section{DISCUSSION}

The lack of an increase in the overall perception of a safety culture, as measured by the surveys, may be attributed to the responses for specific safety domains. In particular, positive responses decreased for the 2 domains of hospital management support and hospital handoffs and transitions, with the latter domain being one of the lowest-ranking domains both before and after CUSP.

The decrease in positive perception of hospital management support reflects the perceived delay in implementation of systemwide changes that were discussed during the safety rounds. Hence, one lesson learned is the need to educate pharmacists that changes at the corporate level may take time, because other departments, disciplines, and stakeholders may have to be involved. The need for involvement of other stakeholders may also explain the post-implementation decrease in perception of the domain of teamwork across units. In other words, we can influence our own practice sooner and more effectively than we can influence the practice of other disciplines. 


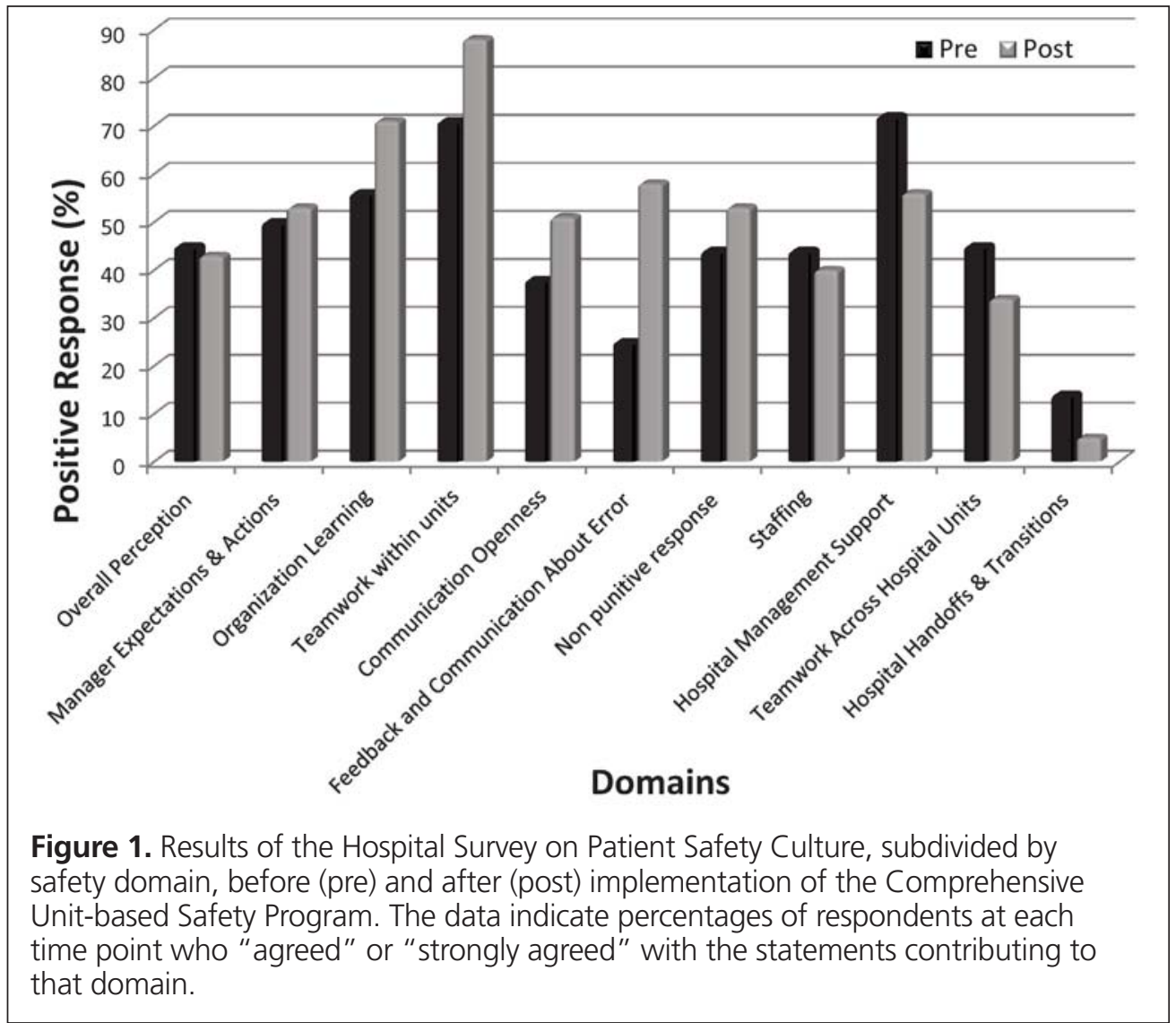

The decrease in positive responses for the perception of hospital handoffs and transitions may be related to the hybrid paper and electronic medication ordering system that was in place as CPOE was implemented over a period of 12 months. Transitions of patients between units with different systems resulted in a large number of errors. Although this transfer-related problem could not be resolved during the safety rounds, discussion increased awareness of the problem and alerted pharmacists to the high potential for errors during patient transfers within the dual system while it was in place.

With the collaborative, interdepartmental discussions initiated through this project, safety rounds brought about increases in positive perceptions for the domains of organizational learning, teamwork within the unit (e.g., within the Department of Pharmacy), and feedback about errors. All of these domains were directly addressed by the safety rounds.

\section{IMPLICATIONS}

Safety rounds were thought by the pharmacists to be valuable and hence were continued beyond the CUSP project period. Since implementation of $\mathrm{CPOE}$ throughout the entire hospital, the type of errors discussed during safety rounds has changed from errors and incidents related to $\mathrm{CPOE}$ transitions of care to medication- specific issues (e.g., tapering doses for steroids). One of the lessons learned was the need to create a pharmacist work group to further evaluate and assess the feasibility of ideas proposed during safety rounds. Recommendations from this work group are discussed during monthly pharmacists' meetings and then implemented. This work group has also been able to create a mechanism for communication of information to other disciplines to address multidisciplinary medication issues.

Although for the purposes of this project, the safety rounds were initially limited to pharmacists, monthly safety rounds have since been implemented for pharmacy technicians. Joint pharmacist and technician safety rounds have also been piloted, with the aim of holding these sessions quarterly, to discuss errors and concerns that affect both groups.

\section{LIMITATIONS}

The project, which led to significant changes in pharmacists' day-to-day activities, was carried out during the summer months, at a time when the department had a large number of staffing changes. This turnover occasionally affected attendance at the safety rounds and may also have affected the response rate for the second survey. Existing practices, policies, and culture at other institutions may limit the generalizability of these results. 


\section{SIGNIFICANCE FOR PRACTICE}

In an increasingly complex medication system, it is important for pharmacists to have a safe venue to discuss and resolve identified safety concerns. At St Michael's Hospital, safety rounds have proven to be an effective mechanism to engage staff, creating a forum where staff can discuss errors and possible solutions to avoid recurrence. Although the measured perception of a culture of safety did not increase during this program, the actions of the pharmacists have improved. Whereas initially errors were reluctantly reported, now both errors and near misses are routinely reported. With pharmacists' ongoing participation in error reporting and the continuation of these safety rounds, we hope that future surveys will reveal an enhanced culture of safety in the Department of Pharmacy of St Michael's Hospital.

\section{References}

1. Pronovost P, Weast B, Rosenstein B, Sexton JB, Holzmueller CG, Paine L, et al. Implementing and validating a comprehensive unit-based safety program. J Patient Saf. 2005;1(1):33-40.

2. Sorra JS, Nieva VF. Hospital survey on patient safety culture. AHRQ Publ No. 04-0041 (prepared by Westat, under Contract No. 290-96-0004). Rockville (MD): Agency for Healthcare Research and Quality; 2004 Sep [cited 2018 Nov 23]. Available from: https://web.patientsafetygroup.org/program/ media/hospcult_guide.pdf

3. Wong E, Chen J, Blackwell, L, Rajakumar I, Parker N, Bombassaro AM. Pharmacy improving patient safety: a retrospective analysis [abstract]. Can J Hosp Pharm. 2011;64(1):70.
Heather Kertland, BScPhm, PharmD, FCSHP, is with the Department of Pharmacy, St Michael's Hospital, and the Leslie Dan Faculty of Pharmacy, University of Toronto, Toronto, Ontario.

Salma Satchu, BScPhm, PharmD, ACPR, is with the Department of Pharmacy, St Michael's Hospital, Toronto, Ontario.

Clarence Chant, BScPhm, PharmD, ACPR, FCSHP, is with the Department of Pharmacy, St Michael's Hospital, and the Leslie Dan Faculty of Pharmacy, University of Toronto, Toronto, Ontario. He is also an Associate Editor with the Canadian Journal of Hospital Pharmacy.

Jill Garland, BScPhm, MSc, is with the Department of Pharmacy, St Michael's Hospital, Toronto, Ontario.

Elaine Tom, BScPhm, is with the Department of Pharmacy, St Michael's Hospital, Toronto, Ontario.

Competing interests: None declared.

Address correspondence to:

Dr Heather Kertland

Department of Pharmacy

St Michael's Hospital

30 Bond Street

Toronto ON M5B 1W8

e-mail: kertlandh@smh.ca

Funding: None received. 